\title{
COMPARATIVE STUDY ON INTRAMEDULLARY NAILING VERSUS AO EXTERNAL FIXATION IN THE MANAGEMENT OF GUSTILO TYPE II, IIIA, AND IIIB TIBIAL SHAFT FRACTURES.
}

1. MBBS, MS (Orthopaedics Surgery) Senior Registrar Orthopaedic Bolan University of Medical \& Health Sciences, Quetta.

2. MBBS, MS (Orthopaedics Surgery) Assistant Professor Orthopaedic Liaquat University of Medical \& Health Sciences Jamshoror / Hyderabad.

3. MBBS, FCPS (Orthopaedics Surgery)

Senior Registrar Orthopaedic Bolan University of Medical \& Health Sciences, Quetta.

4. MBBS, MS (Orthopaedics Surgery) Orthopedic Surgeon

Sindh Government Taluka Hospital, Thano Bula Khan, Jamshoro.

5. MBBS, MS (Orthopaedics)

Senior Registrar Orthopaedics

Peoples University of Medical \& Health Sciences for Women Shaheed Benazirabad.

6. MBBS, MS (General Surgery) Resident General Surgery Liaquat University of Medical \& Health Sciences Jamshoror / Hyderabad.

Correspondence Address: Dr. Khawand Bukhsh Umrani Department of Orthopaedic

Bolan University of Medical \& Health Sciences Quetta. farooq346@gmail.com

Article received on:

31/08/2019

Accepted for publication:

29/02/2020

\section{INTRODUCTION}

Most of the complications of open fractures of the tibia are difficult to handle by any of an established form of treatment. Open fractures of the tibia are the most common open long bone fractures, with an annual incidence of 3.4 per $100000 .^{1}$

All accessible techniques can be used to accomplish stability in Grade II wounds with appropriate soft tissue cover.

These include intramedullary devices; rigid or flexible inner plating devices inserted by biological method, reamed orunreamed; external fixators and a mixture of the above. ${ }^{2}$ Skeletal traction entails some serious hazards because
Khawand Bukhsh Umrani' ${ }^{1}$ Zamir Hussain Tunio² ${ }^{2}$ Mohammad Aslam Mengal ${ }^{3}$, Abdul Hafeez Qureshi ${ }^{4}$, Parvez Ahmed ${ }^{5}$, Naimatullah Kalhoro ${ }^{6}$

ABSTRACT... Objectives: To compare the external fixator and unreamed interlocking Sciences Jamshoro/Hyderabad. Period: Two years February 2016 to January 2018. Material \& ( were treated by unreamed solid interlocking nail. A total of 14 cases, that is, $31 \%$ showed delayed union. Type II injury $3 / 15=20 \%$; Type IIIA injury $6 / 22=27.3 \%$; Type IIIB injury it is $-5 / 8$ was $13.6 \%$, and external fixation was $8.7 \%$. Reoperation in the form of dynamization and are a good and reliable method of stabilization of Gustilo type II, type IIIA, and type III tibial diaphyseal fractures, unreamed interlocking intramedullary nail yields better results in treating Gustilo type II and type IIIA tibial shaft fractures.

Key words: $\quad$ External Fixation, Intramedullary Nailing, Open Fractures, Tibial Diaphysis.

Article Citation: Umrani KB, Tunio ZH, Mengal MA, Qureshi AH, Ahmed P, Kalhoro N. Comparative study on intramedullary nailing versus $A O$ external fixation in the management of gustilo type II, IIIA, and IIIB tibial shaft fractures. Professional Med J 2020; 27(6):1199-1205.

DOI: 10.29309/TPMJ/2020.27.06.4096

any traction allows motion at the fracture site, what so ever well-balanced traction may be. For type III fractures, where soft tissue coverage is not possible, only intramedullary device and external fixation are the options. ${ }^{3-8}$

The use of External fixation in Gustilo type III fractures may offer many advantages. It is the manipulation, alignment, and stabilization of bone structures with pins, wires, and screws or other bone fasteners that attach the bone to an external scaffold or frame. ${ }^{2}$ The main supporting frame is attached to the ends of pins and therefore leaving room for dressing or procedures such as skin grafting or other soft tissue procedure. It can be used quickly for stabilization in a patient 
with shock. It is having many advantages like rigid fixation and good access for wound care, wide space for secondary soft tissue procedure, maintains the limb length, helpful in segmental fractures and any angulation, overlapping, displacement or rotation of fragments can be corrected. No special operation theater or equipment is needed. ${ }^{9-13}$

In recent years, the use of the unreamed intramedullary locked apparatus for open fractures grade II and grade III has achieved prominence. Several comparative studies conclude that it is at least as efficient if not more efficient than using an external fixator and that it has an acceptable level of complication. It gives better stability and promises early weight-bearing. Therefore, in grade III tibial fractures the decision making is based on the wound condition, the amount of muscle damage, type of fractures, and availability of equipment ..$^{7-9,11,12}$ Hence, we performed a comparative study between external fixator and recently popularized unreamed interlocking nail in treating Gustilo type II, type IIIA, and type IIIB tibial diaphyseal fractures to come to a conclusion to establish their appropriateness in the respective fractures.

\section{MATERIAL \& METHODS}

This randomized control trial study was conducted on 45 cases of open tibia fractures in the department of Orthopaedic Surgery and Traumatology (DOST) LiaquatUniversity ofMedical and Health Sciences Jamshoro/Hyderabad for the period of two years February 2016 to January 2018. Patients with open diaphyseal fracture of tibia who were Gustilo type II, type IIIA, and type IIIB tibial diaphyseal fractures, arriving within $6 / 24$ hours of injury and age range was $20-50$ years (Male and female) were included in the study whereas patients with diaphyseal fractures with intraarticular extension, More than 12 hold injuries, Associated systemic injuries (e.g., head injury, abdominal injury), Associated co-morbid conditions such as diabetes mellitus, ischemic heart disease, chronic obstructive pulmonary disease, Associated other major fractures (e.g., hip fractures, spine fractures) were not included.
Open fractures of the tibia are frequently associated with other major injuries. The standard protocol of the "Advanced Trauma Life Support" program was maintained.

On admission, a rapid survey was done. Emergency measures are taken to combat pain, hemorrhage, and shock with proper sedation, analgesic, intravenous infusion or transfusion of blood when required.

The examination includes determining the extent and type of soft tissue wound and whether there are any vascular and nerve damage. Immediate treatment of vascular injury or compartment syndrome for limb salvage and avoiding tissue ischemia can result in irreversible muscle and nerve damage if present for 8 hours or longer. Two swabs were taken from the wound (one from superficial and other from deep part) and sent for culture sensitivity. A high dose of broad-spectrum bactericidal antibiotics was administered.

Swabs for the culture of the wounds were taken prior to any irrigation and administration of antibiotics. Shaving was done surrounding the wound and preliminary skin preparation with savlon and povidone-iodine was carried out. All foreign materials embedded within wounds were removed meticulously, and the wound was thoroughly irrigated with a copious amount of normal saline. Considerable efforts were made to minimize soft tissue trauma.

We had done thorough debridement under general anesthesia within $6 \mathrm{~h}$ of admission.

We initiated a methodical, layer by layer debridement beginning from the skin and subcutaneous tissue. A margin of healthy skin, as minimum as less from the contaminated and contused skin border, was excised. Debridement continued until all the devitalized tissue had been removed layer by layer to the depth of the wound but respecting the integrity of important structures such as blood vessels, nerves, and tendons. All foreign materials were removed either by washing or by excision of the tissue. 
Antibiotics were started intravenously before surgery and continued for 2 weeks of the postoperative period routinely and further extended depending upon the status of the wound and culture sensitivity report.

Following debridement, the wound was covered with a sterile dressing soaked with normal saline and pressure bandages were applied. The fractures splinted by a temporary POP back slab with the achievement of reduction as much as possible and planning done for operation.

The choice of fixation devices, the open fracture care of the two treatment groups was similar. All patients received emergency irrigation and debridement as well as swab sensitivity, accompanied by bone stability.

After proper open reduction and holding the fracture, the most distal Schanz screw was introduced near to the ankle joint through the "Safe Soft Tissue corridor" which lies anteromedially and varies in size from an arc $220^{\circ}$ close to the tibial plateau to $120^{\circ}$ just above the ankle joint. By insertion of pins through this safe corridor, injury to the main vessels, nerves, and musculotendinous units was avoided.

After inserting the Schanz screw, drill sleeve was removed, and a universal clamp was attached to the Schanz screw and was fixed to it approximately $40 \mathrm{~mm}$ from the surface of the tibia.

Now, an $11 \mathrm{~mm}$ tube of appropriate length was attached to these clamps so that it was posterior to the Schanz screw. Three universal clamps were sliding into this tube and were fixed to the tube at predetermined sites of Schanz screw insertion.

Most proximal Schanz screw was then introduced below the knee joint through the most proximal clamps using the technique as described before, after ensuring that it was parallel to the first screw in both planes and then it was fixed to the clamp.

The construction of the mirror image, that is, uniplanar and unilateral frame or delta frame (biplanar) was done according to the requirements. Now along the anteromedial aspect of the tibia a second single tube unilateral frame was constructed at an angle of $60-90^{\circ}$ to the first frame. Preload was applied to the Schanz screw of each frame. Two subframes were connected with a pair of short tubes using Tube to Tube clamps. Using universal clamps and connecting rods or Steinmann pin could also connect this. This connection enhances the rotational stability of the assembly. The patients were followed up at every 4 weeks interval. They were examined clinically and radiologically. Patients were advised to do quadriceps exercises, active straight leg raise and knee bending exercises since 1st postoperative day. If, after good healing of the soft tissues, check X-ray showed sufficient callus for union and the patient walked without support having no pain, we thought regarding the removal of external fixation.

If the patient had knee or ankle stiffness, local ultrasonic therapy with a wax bath was advised for 10-14 days to avoid muscle spasm. After 3-6 months external fixator was removed followed by PTB cast for 4-8 weeks and weight-bearing was progressively increased in a customized manner. As usual, patients were regularly checked both clinically and radiologically until the complete union is achieved in the follow-up period. Then the plaster was removed, and the patients were rehabilitated to normal activities gradually.

The arbitrary standard considered for the time of union, delayed union, and nonunion were as follows:

Normal union - union occurring before 30 weeks

- Delayed union - union occurring after 30 weeks

- Nonunion - no union even after 9 months.

The fracture was considered healed when:

- It was clinically stable.

- The roentgenogram showed sufficient callus for weight-bearing.

- The patient was able to walk without discomfort and support.

\section{Data Analysis}

All the data analysis was done using the SPSS 
program. Frequency and percentage were calculated for categorical variables such as gender, mode of injury, wound condition at the time of arrival, Gustilo classification (GII \& GIIIA, GIIIB), range of movement and complications were presented as $\mathrm{n}(\%)$ and Chi-square test was applied to compare the proportions. Numerical data like age (in years), time between injury and arrival procedure (hour), time between arrival \& primary procedure (hour), time between primary procedure \& fixation, time of full weight-bearing, time of dynamization, time of union and hospital stay (in days) were expressed as mean + standard deviation and student t-test (2 tailed) was applied to compare the means among the both groups. A $p$-value $<0.05$ was considered as the statistically significant level for all the comparisons.

\section{RESULTS}

In this series of 45 cases of open tibial diaphyseal fractures (Gustilo type II, IIIA, IIIB) had been studied. Of 45 cases, 23 cases were treated by external fixator and 22 cases were treated by an unnamed solid interlocking nail. The choice of the fixator was selected randomly for an approximately equal distribution in each group of cases. Table-I shows the overall results.

For type II and type IIIA injury undreamed interlocking nail yielded a better result.

For type IIIB injury, no implant showed superior to the other as far as the union is concerned.

The rate of the union in type IIIA fractures with unreamed interlocking nail was higher, probably due to more stable fixation and early weightbearing.

A total of 14 cases, that is, $31 \%$ showed delayed union. Type II injury $3 / 15=20 \%$; Type IIIA injury $6 / 22=27.3 \%$; Type IIIB injury it is $-5 / 8=12.5 \%$. For cases treated with unreamed nail, it was $22.7 \%$; the external fixator was $39.1 \%$.

Among 45 cases, 05 cases showed nonunion. For cases treated with unreamed nail, it was $13.6 \%$; external fixation was $8.7 \%$. The overall rate of deep infection is $11.1 \%$. The rate in interlocking nail was $13.6 \%$, and external fixation was $8.7 \%$. About $17.8 \%$ of patients developed ankle stiffness. The rate of ankle stiffness in unreamed nails is $9.1 \%$, and external fixation is $26.1 \%$. The overall rate of malunion with an unreamed nail is $13.6 \%$, and external fixation is $26.1 \%$. Reoperation in the form of dynamization and bone graft application after 20 weeks in unreamed nail was $36.4 \%$, whereas in external fixation the rate was high, almost $48 \%$. The average time of union with unreamed interlocking was about 25.4 weeks, wherein external fixation was 28.6 weeks. In our series only in 17 cases, wounds healed by secondary intention and 12 cases needed delaying primary closure. In the rest of the cases (16), we have to perform different late reconstructive procedures such as split-thickness skin grafting, gastrocnemius myocutaneous flap, and soleus flap application. Six (13.3\%) patients had necrosis of the margins noticed when the dressing was opened for the 1st time. All healed by secondary intention with usual treatment within 3-4 weeks' time.

Twelve (26.6\%) cases required delayed primary suture for wound closure. Wounds were covered with gauze soaked in saline to prevent drying of the tissues. The dressing was changed on every alternate day till delayed stitches done on the 4-5th postoperative day. The wound healed without further complications. Twelve (26.7\%) had serious discharge from the wound. Four cases had a negative culture. The culture mostly showed Staphylococcus aureus and only two cases showed positive Pseudomonas infection. They were treated by antibiotics according to sensitivity and regular dressing. Only two cases continued to drain through a small sinus, but later that discharge was culture negative. Ten patients (22.2\%) developed a superficial infection (mostly culture-positive S. aureus). With unreamed nail, it was $18.2 \%$ and with external fixator $-26.1 \%$. Five patients $(11.1 \%)$ developed a deep wound infection. The culture showed mostly S. aureus or Pseudomonas. One case was Klebsiella culture positive.

Of 45 patients, one patient developed weakness of dorsiflexion of the ankle on the next postoperative day. The Schanz screw was taken out and again reintroduced in the separate site. The neurological 
deficit gradually recovered.

Three patients showed a gross restriction of ankle movements during the postoperative days. In two cases of the interlocking nail, there was an avulsion fracture of the tibial tuberosity. Seven $(30 \%)$ out of twenty-three patients who had undergone external fixator developed pin tract infections. Ankle stiffness developed in 8 (17.8\%) cases. Of these 5 cases, there were $<25 \%$ and in the other 3 cases, there was more than $25 \%$ stiffness. The patients are having $<25 \%$ of the stiffness had no difficulty in walking but squatting was slightly difficult for them.

Nine (20\%) developed malunion more with external fixation. Six patients developed angulation in various directions varying from $5^{\circ}$ to $10^{\circ}$. Five patients had $10-15^{\circ}$ rotational deformity. Six $(13.3 \%)$ patients showed shortening varying from $0.5 \mathrm{~cm}$ to $2 \mathrm{~cm}$ in those severely comminuted and unstable fractures. None had any serious problem due to this shortening.

Ten patients out of 22 cases of interlocking nail developed anterior knee pain. Nine patients (20\%) developed knee stiffness in the postoperative days. Totally, 25 patients (56\%) out of 45 showed thigh muscle wasting from $1 \mathrm{~cm}$ to $4 \mathrm{~cm}$ in comparison to the healthy side. More wasting was seen in cases of external fixation. Screw breakage occurred in 3 cases (13.6\%) out of 32 unreamed interlocking nails.

\section{DISCUSSION}

In the present series, a comparative study was done to evaluate the role of external fixation and unreamed interlocking nail in the treatment of open fractures tibia (Gustilo type II, type IIIA, and type IIIB) and to compare the results obtained after treating these cases by the respective methods considering different parameters.

According to Fang et al. in $2012^{3}$ who selected 9 research papers for meta-analysis from medical literature databases. Of these, malunion and deep infection rate, nonunion, and comparison of time to the union were included. He found that external fixation had a higher malunion rate as compared to unreamed intramedullary nailing. the study conducted by Xue et al. in $2014^{14}$ included 842 patients out of 6620 , to determine that in the treatment of DTF, which method is better such as plating or IMN for complications. ${ }^{14}$ He found that IMN method was better with low rate complications and infection, timely union but the plating was found to lower malunion than IMN $(p$-value $=<0.00001)$

In 2011, Mohseni et al. ${ }^{15}$ compared OA tubular external fixation in open grade IIIA $\square$ IIIB tibial shaft fractures with unreamed intramedullary nailing. The time of union was $3,4,5$, and $6<$ or $=$ weeks after operation in $28,12,32$, and $28 \%$ of the cases in unreamed intramedullary group versus 4, 12, 48 , and $36 \%$ of the cases in external fixation group, respectively. There were 16, 8, 0, and $4 \%$ cases of infection, soft tissue injury, malunion and non-union after the procedure of undreamed intramedullary group as compared to $32,12,24$, and $8 \%$ cases of external fixation group $(P=0.19$, $0.50,0.02$, and 0.50 , respectively).

\begin{tabular}{|c|c|c|c|c|}
\hline & $\begin{array}{l}\text { Unreamed Interlocking Nail } \\
\qquad n=22(\%)\end{array}$ & $\begin{array}{l}\text { External Fixation } \\
\qquad n=23(\%)\end{array}$ & $\begin{array}{c}\text { Total } \\
n=45(\%)\end{array}$ & P-Value \\
\hline Normal union & $14(63.6 \%)$ & 12 (52.2\%) & $26(57.8 \%)$ & $>0.05$ \\
\hline Nonunion & $3(13.6 \%)$ & $2(8.7 \%)$ & $5(11.1 \%)$ & $<0.05$ \\
\hline Superficial infection & $4(18.2 \%)$ & $6(26.1 \%)$ & $10(22.2 \%)$ & $<0.05$ \\
\hline Mal union & $3(13.6 \%)$ & $6(26.1 \%)$ & $9(20.0 \%)$ & $<0.05$ \\
\hline Reoperation & $8(36.4 \%)$ & $11(47.9 \%)$ & $19(42.2 \%)$ & $>0.05$ \\
\hline Time of union (weeks) & 25.4 & 28.6 & 27.1 & $<0.05$ \\
\hline
\end{tabular}


The mean ambulation time after the operation was $2.92 \pm 2.43$ weeks in the unreamed intramedullary nailing group vs. $2.68 \pm 2.14$ weeks in the external fixation group $(P=0.71)$.

There was a total of 486 patients of tibial fractures in the study of Metsemakers et al. ${ }^{16}$ in which, delayed union and nonunion, including fracture type, open fractures, and Gustilo type were similar risk factors analyzed by Univariate regression.

Nonunion occurred due to a single risk factor of polytrauma calculated by multiple logistic regression analysis.

Reamed tibial nails (RTNs) have been correlated with considerably greater early union odds relative to unreamed tibial nails (UTNs) in open fractures of IIIB in research done by Papakostidis et al. ${ }^{17}$ For both therapy methods (RTN, UTN), significantly increased profound infection rates of open IIIB fractures relative to all other grades have been recorded.

Compared to grades I and II, lower profound infection rates for IIIA open fractures treated with RTNs were reported.

Forty-two open tibial fractures were treated with secondary IMN after EF by Yokoyama et al. ${ }^{10}$ Seven $(16.7 \%)$ of the 42 open tibia fractures developed deep infections. All deep infections occurred in Gustilo type III $(22.6 \%, 7 / 31)$. Only the skin closure time was a significant factor affecting the occurrence of deep infection on the present analysis $(P=0.006)$.

Noumi et al. ${ }^{18}$ in 2005 examined 89 open femoral fractures treated with immediate or delayed locked IMN in a static fashion. Multivariate analysis revealed that only Gustilo type significantly correlated with the occurrence of deep infection $(P<0.05)$; only fracture grade by $A O$ type significantly correlated with the occurrence of nonunion $(P<0.02){ }^{18}$

Results of the present study suggest that though external fixators are good and reliable methods of stabilization of Gustilo type II, type IIIA, and type IIIB tibial diaphyseal fractures, unreamed interlocking intramedullary nail yields better results in treating Gustilo type II and type IIIA tibial shaft fractures. The question of whether Gustilo type III tibial fractures should be treated with non-reamed IMN or EF was addressed in a previous meta-analysis ${ }^{19}$, where Fang et al. highlighted a lower malunion rate with non- reamed IMN and no significant differences in deep infection, non-union and time to union. They included prospective, randomized, case-control and cohort studies and examined the deep infection, malunion, non-union and time to union. Their meta-analysis did not highlight clear advantages for either approach except in relation to the malunion rate, which, however, is not a key factor determining treatment selection. We included only RCT describing IMN and EF and assessing infections, fracture healing problems and "other complications." 19

\section{CONCLUSION}

Results suggest that though external fixators are a good and reliable method of stabilization of Gustilo type II, type IIIA, and type IIIB tibial diaphyseal fractures, unreamed interlocking intramedullary nail yields better results in treating Gustilo type II and type IIIA tibial shaft fractures.

\section{Limitation of the Study}

There is no limitation of this study.

\section{Acknowledgment}

We are thankful to Farooq Ahmed Mangnejo and Mr. Saddam Berahmani Balouch for statistical analysis and other technical help in the manuscript.

Copyright $\odot 29$ Feb, 2020.

\section{REFERENCES}

1. Elniel AR, Giannoudis $P$ V. Open fractures of the lower extremity. EFORT Open Rev. 2018 May; 3(5):316-25.

2. Chaudhuri A, Datta S, Ghosh P, Patil D, Sinha S, Ghosh $S$. Comparative studies on intramedullary nailing versus ao external fixation in the management of gustilo type II, IIIA, and IIIB tibial shaft fractures. Saudi J Sports Med. 2015; 15(3):262.

3. Fang X, Jiang L, Wang Y, Zhao L. Treatment of Gustilo grade III tibial fractures with unreamed intramedullary nailing versus external fixator: A meta-analysis. Med Sci Monit. 2012; 18(4):RA49-RA56.

4. Kakar S, Tornetta P. Open fractures of the tibia treated by immediate intramedullary tibial nail insertion without reaming: A prospective study. J Orthop Trauma. 2007 Mar; 21(3):153-7.

5. Jiang C, Tang MJ SZ. Treatment of open tibial fractures: Comparison of non-reamed interlocking intramedullary nail and external fixator. China $\mathrm{J}$ Orthop Trauma. 2000; 13:14-5. 
6. Kaftandziev I, Pejkova S, Saveski J. Operative treatment of III grade open fractures of the tibial diaphysis. Prilozi. 2006 Jul; 27(1):121-31.

7. Bibbo C, Brueggeman J. Prevention and management of complications arising from external fixation pin sites. J Foot Ankle Surg. 2010 Jan; 49(1):87-92.

8. Lam SW, Teraa M, Leenen LPH, van der Heijden GJMG. Systematic review shows lowered risk of nonunion after reamed nailing in patients with closed tibial shaft fractures. Injury. $2010 \mathrm{Jul} ; 41(7): 671-5$.

9. Yokoyama K, Itoman M, Uchino M, Fukushima K, Nitta $\mathrm{H}$, Kojima Y. Immediate versus delayed intramedullary nailing for open fractures of the tibial shaft: $A$ multivariate analysis of factors affecting deep infection and fracture healing. Indian J Orthop. 2008; $42(4): 410$.

10. Yokoyama K, Uchino M, Nakamura K, Ohtsuka H, Suzuki T, Boku T, et al. Risk factors for deep infection in secondary intramedullary nailing after external fixation for open tibial fractures. Injury. 2006 Jun; $37(6): 554-60$.

11. Ziran B, Darowish M, Klatt BA, Agudelo JF, Smith WR. Intramedullary nailing in open tibia fractures: $A$ comparison of two techniques. Int Orthop. 2004 Aug $25 ; 28(4)$.

12. Okike $\mathrm{K}$, Bhattacharyya $\mathrm{T}$. Trends in the management of open fractures. J Bone Jt Surg. $2006 \mathrm{Dec}$; 88(12):2739-48.

13. Bhandari $M$, Zlowodzki $M$, Tornetta $P$, Schmidt $A$, Templeman DC. Intramedullary nailing following external fixation in femoral and tibial shaft fractures. J Orthop Trauma. 2005 Feb; 19(2):140-4.
14. Xue X-H, Yan S-G, Cai X-Z, Shi M-M, Lin T. Intramedullary nailing versus plating for extra-articular distal tibial metaphyseal fracture: A systematic review and metaanalysis. Injury. $2014 \mathrm{Apr}$; 45(4):667-76.

15. Mohseni MA, Soleimanpour J, Mohammadpour $H$, Shahsavari A. AO tubular external fixation vs. unreamed intramedullary nailing in open grade IIIAIIIB tibial shaft fractures: A single-center randomized clinical trial. Pakistan J Biol Sci PJBS. 2011 Apr 15; 14(8):490-5.

16. Metsemakers W-J, Handojo K, Reynders P, Sermon A, Vanderschot $\mathrm{P}$, Nijs $\mathrm{S}$. Individual risk factors for deep infection and compromised fracture healing after intramedullary nailing of tibial shaft fractures: A single centre experience of $\mathbf{4 8 0}$ patients. Injury. 2015 Apr; 46(4):740-5.

17. Papakostidis C, Kanakaris NK, Pretel J, Faour O, Morell DJ, Giannoudis P V. Prevalence of complications of open tibial shaft fractures stratified as per the Gustilo-Anderson classification. Injury. 2011 Dec 42(12):1408-15.

18. Noumi T, Yokoyama K, Ohtsuka H, Nakamura K, Itoman M. Intramedullary nailing for open fractures of the femoral shaft: evaluation of contributing factors on deep infection and nonunion using multivariate analysis. Injury. 2005 Sep; 36(9):1085-93.

19. Giovannini F, de Palma L, Panfighi A, Marinelli M. Intramedullary nailing versus external fixation in Gustilo type III open tibial shaft fractures: A metaanalysis of randomized controlled trials. Strategies Trauma Limb Reconstr. 2016 Apr 30; 11(1):1-4.

\begin{tabular}{|c|l|l|}
\hline \multicolumn{2}{|c|}{ AUTHORSHIP } & AND CONTRIBUTION DECLARATION \\
\hline Sr. \# & \multicolumn{1}{|c|}{ Author(s) Full Name } & \multicolumn{1}{|c|}{ Contribution to the paper } \\
\hline 1 & $\begin{array}{l}\text { Khawand Bukhsh } \\
\text { Umrani }\end{array}$ & $\begin{array}{l}\text { Designed the research, assessed the vases, } \\
\text { wrote the paper, Interpretation of discussion } \\
\text { and data entry in SPSS. } \\
\text { Collected the data, did the literature search, } \\
\text { drafted the manuscript assisted in writing } \\
\text { the paper. } \\
\text { Involved in data collection, analyzed the } \\
\text { data revised the manuscript. } \\
\text { Revised the original manuscript, reviewed } \\
\text { the cases, analyzed the data and assisted } \\
\text { in writing the paper, Interpretation in results } \\
\text { writing. } \\
\text { References, citation manager \& designing } \\
\text { of results and charts and Graphs in } \\
\text { manuscript. } \\
\text { Data entry in SPSS and other technical } \\
\text { help, help in corrections. }\end{array}$ \\
\hline 5 & $\begin{array}{l}\text { Zamir Hussain Tunio } \\
\text { Mohammad Aslam }\end{array}$ \\
\hline 6 & Abdul Hafeez Quresh \\
\hline Parvez Ahmed & Naimatullah Kalhoro \\
\hline
\end{tabular}

